\title{
Active vibration control of piezoelectric plates
}

\author{
Marwan Abukhaled ${ }^{\mathrm{a}}$ \\ Department of Mathematics and Statistics, American University of Sharjah, Sharjah, United Arab Emirates
}

\begin{abstract}
The control of thermally induced vibrations of a rectangular plate is investigated. An optimization problem is formulated to determine the control voltage needed to perform vibration suppression with least control effort. By eigenfunction expansion, the optimal control problem will be converted from a distributed to a lumped parameter system. By utilizing the variational theory, an explicit optimal control criterion will be derived.
\end{abstract}

\section{Introduction}

The dynamics and the stability of a structure are likely to be effected as a result of rapid temperature variation. The change in temperature experienced by a spacecraft emerging from a shadow entering the sunlight is an example of a dynamic load experienced by a structure. The sudden exposure to heat leads to thermal vibrations, which needs to be damped. Structures subject to thermally induced vibrations have been discussed by many authors. For example, the piezo thermoelastic effects of distributed piezoelectric sensors and actuators in higher dimension structures such as plates and shells are discussed in $[?, ?, ?, ?]$. Sadek et al [?], discussed vibrations suppression of a thermoelastic beam subject to sudden heat input. A thermoelastic beam controlled by an active control exercised by point actuators is examined in [?]. Friswell et al discussed a closed loop control to suppress the vibrations of peizo thermoelastic beam [?]. In [?], numerical studies are presented for thermally induced vibrations of peizo-thermo-viscoelastic composite beam subjected to a transient thermal load using coupled finite element method.

In this paper, we consider a three-layer plate consisting of a thermal host layer and two outer piezothermoelastic layers as in Figure 1.

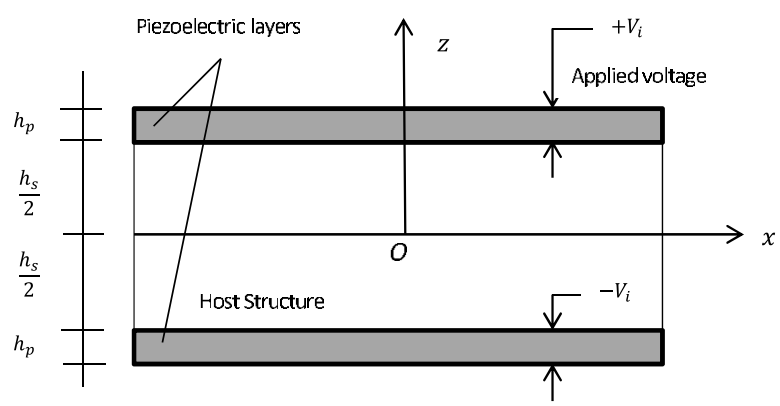

Fig. 1. A piezoelectric sandwich plate.

The plate is assumed to be exposed to a sudden rise in temperature over one face, which will cause an undesired vibration. The main objective of this research article is to

\footnotetext{
a e-mail: mabukhaled@aus.edu
}

minimize a weighted quadratic functional of the dynamic responses of the smart thin plate in a prescribed terminal time using continuous piezoelectric patches (voltages $\pm V_{i}$ ), with the least possible expenditure of control forces. The solution method is a combination of modal expansion and variational approaches. The modal expansion approach is used to eliminate the space parameter and hence convert the problem form an optimal control of a distributed parameter system to an optimal control of a lumped parameter system. The variational approach will be employed to derive an explicit optimal control criterion. The resulting set of ordinary differential equations will be solved to determine the corresponding displacement and velocity.

\section{Optimal control problem}

The equation of motion governing the non dimensional regular plate is given by

$$
\mu w_{t t}+D \nabla^{4}\left(w+2 \zeta w_{t}\right)=-\Gamma_{1} v(t) \nabla^{2}[\Delta H(x, y)],
$$

where

$$
\Delta H(x, y)=\left[H\left(x-x_{1}\right)-H\left(x-x_{2}\right)\right] \times\left[H\left(y-y_{1}\right)-H\left(y-y_{2}\right)\right] .
$$

Here $\zeta$ denotes the strain-rate damping coefficient, $D$ denotes the effective flexural stiffness, $\mu$ is mass per unit area, $\Gamma_{1}$ is the electric moment due to electric field applied to piezoelectric layers, $v(t)$ is the applied voltage to the piezoelectric patch (see [?] and the references therein for further details). The boundary conditions for the simply supported plate are given by

$$
\left.w(x, y, t)\right|_{\partial \Omega}=0,\left.\quad D \nabla^{2} w(x, y, t)\right|_{\partial \Omega}=0,
$$

where $\Omega=\{(x, y): 0 \leq x \leq a, 0 \leq y \leq b\}$ and $\partial \Omega=$ $\{(x, y): x=0, a$ and $y=0, b\}$. The initial conditions are given by

$$
w(x, y, 0)=w^{0}(x, y), \quad w_{t}(x, y, 0)=w^{1}(x, y) .
$$

Let $U_{a d}=v(t): v(t) \in L^{2}\left(\Omega_{t}\right)$, where $\Omega_{t}=\left(0, t_{f}\right)$ be the set of admissible controls for a given terminal time $t_{f}$. 
Consider the performance index

$$
\begin{aligned}
J[v(t)]= & \iint_{\Omega}\left(\mu_{1} w^{2}\left(x, y, t_{f}\right)+\mu_{2} w_{t}^{2}\left(x, y, t_{f}\right)\right) d x d y \\
& +\int_{0}^{t_{f}} \mu_{3} v^{2}(t) d t
\end{aligned}
$$

where $\mu_{1}, \mu_{2}$, and $\mu_{3}$ are nonnegative weighting factors satisfying $\mu_{1}+\mu_{2} \geq 0$ and $\mu_{3}>0$. The second integral on the right hand side of (4) is a penalty term in the control energy. The optimal control problem is now stated as follows: Determine the optimal control $v^{*}(t) \in U_{a d}$ so that

$$
J\left[v^{*}(t)\right]=\min _{v(t) \in U_{a d}} J[v(t)]
$$

and subject to the governing PDE (1) and the boundary and initial condition (2)-(3).

\section{Control problem in modal space}

Let

$$
u(x, y, t)=w(x, y, t)+\phi(y) \psi(t)
$$

where $\phi(y)=\frac{y^{3}(y-l)}{6 l^{2}}$. Substituting (6) into equation (1) gives

$$
\mu u_{t t}+D \nabla^{4}\left(u+2 \zeta u_{t}\right)=-\Gamma_{1} v(t) \nabla^{2}[\Delta H(x, y)]+\Psi(y, t),
$$

where

$$
\Psi(y, t)=\mu \phi(y) \psi^{\prime \prime}(t)+D\left[\phi(y) \psi(t)+2 \zeta \phi(y) \psi^{\prime}(t)\right]
$$

with new boundary and initial conditions given by

$$
\begin{gathered}
u(x, y, t)=u_{x x}(x, y, t)=0 \text { for } x=0, a \\
u(x, y, t)=u_{y y}(x, y, t)=0 \text { for } y=0, b \\
u(x, y, 0)=w^{0}(x, y)+\phi(y) \psi(0) \\
u_{t}(x, y, 0)=w^{1}(x, y)+\phi(y) \psi^{\prime}(0)
\end{gathered}
$$

and the performance index becomes

$$
\begin{aligned}
J[v(t)]= & \iint_{\Omega}\left(\mu_{1} u^{2}\left(x, y, t_{f}\right)+\mu_{2} u_{t}^{2}\left(x, y, t_{f}\right)\right) d x d y \\
& +\int_{0}^{t_{f}} \mu_{3} v^{2}(t) d t .
\end{aligned}
$$

The optimal control problem is now restated as follows: Determine the optimal control $v^{*}(t) \in U_{a d}$ so that

$$
J\left[v^{*}(t)\right]=\min _{v(t) \in U_{a d}} J[v(t)]
$$

and such that equations (7)-(9) hold. The expansion

$$
u(x, y, t)=\sum_{m=1}^{M} \sum_{n=1}^{N} W_{m n}(t) \varphi_{m n}(x, y)
$$

where

$$
\varphi_{m n}(x, y)=X_{m}(x) Y_{n}(y)
$$

in which

$$
\begin{aligned}
& X_{m}(x)=\sqrt{\frac{2}{a}} \sin \left(\frac{m \pi x}{a}\right) \\
& Y_{n}(y)=\sqrt{\frac{2}{b}} \sin \left(\frac{n \pi y}{b}\right)
\end{aligned}
$$

converts equation (7) to the following set of ordinary differential equations

$$
\begin{aligned}
& \frac{d^{2}}{d t^{2}} W_{m n}+2 \zeta \lambda^{2} \frac{d}{d t} W_{m n}+\lambda_{m n}^{2} W_{m n}=f(t) \\
& \quad+\Gamma_{1} \Gamma_{2} v(t) F_{1}\left(x_{1}, x_{2}\right) F_{2}\left(y_{1}, y_{2}\right)
\end{aligned}
$$

where

$$
\begin{aligned}
\Gamma_{2} & =-\left(\frac{b_{j}}{a_{k}}+\frac{a_{k}}{b_{j}}\right) \frac{2}{\sqrt{a b}} \\
F_{1}\left(x_{1}, x_{2}\right) & =\cos \left(\frac{j \pi x_{2}}{a}\right)-\cos \left(\frac{j \pi x_{1}}{a}\right) \\
F_{2}\left(y_{1}, y_{2}\right) & =\cos \left(\frac{j \pi y_{2}}{b}\right)-\cos \left(\frac{j \pi y_{1}}{b}\right) \\
\lambda_{m n}^{2} & =\frac{D \pi^{4}}{\mu a^{4}}\left(m^{4}+2 m^{2} n^{2} R^{2}+n^{4} R^{4}\right) \\
f(t) & =\iint_{\Omega} \Psi(y, t) \varphi_{m n}(x, y) d x d y
\end{aligned}
$$

in which $R=\frac{a}{b}<1$. The general solution of equation (15) is given by

$$
W_{m n}(t)=W_{m n}^{h}+W_{m n}^{p}
$$

where

$$
\begin{aligned}
W_{m n}^{h}(t)= & e^{-\zeta \lambda_{m n}^{2} t}\left[c_{1} \cos \bar{\lambda}_{m n}(t-\tau)+c_{2} \sin \bar{\lambda}_{m n}(t-\tau)\right] \\
& +\int e^{-\zeta \lambda_{m n}^{2}(t-\tau)} \sin \left(\bar{\lambda}_{m n}(t-\tau)\right) f(\tau) d \tau
\end{aligned}
$$

and

$$
\begin{aligned}
W_{m n}^{p}(t)= & \Gamma_{1} \Gamma_{2} v(t) F_{1}\left(x_{1}, x_{2}\right) F_{2}\left(y_{1}, y_{2}\right) \times \\
& \int e^{-\zeta \lambda_{m n}^{2}(t-\tau)} v(t) \sin \left(\bar{\lambda}_{m n}(t-\tau)\right) f(\tau) d \tau
\end{aligned}
$$

in which $\bar{\lambda}_{m n}=\lambda_{m n}^{2} \sqrt{1-\lambda_{m n}^{2} \zeta^{2}}$. Using initial conditions (9), we obtain

$$
\begin{aligned}
W_{m n}(t)= & e^{-\zeta \lambda_{m n}^{2} t}\left[c_{1} \cos \bar{\lambda}_{m n}(t-\tau)+c_{2} \sin \bar{\lambda}_{m n}(t-\tau)\right]+ \\
& +\frac{A_{m n}}{\bar{\lambda}_{m n}} \int_{0}^{t_{f}} e^{-\zeta \lambda_{m n}^{2} t} \sin \left(\bar{\lambda}_{m n}(t-\tau) \sum_{j=1}^{\infty} e^{-\gamma_{j}^{2} t} d \tau+\right. \\
& +\Psi \int_{0}^{t_{f}} e^{-\zeta \lambda_{m n}^{2} t} \sin \left(\bar{\lambda}_{m n}(t-\tau) v(\tau) d \tau\right.
\end{aligned}
$$

where

$$
\begin{aligned}
& c_{1}=\frac{1}{\bar{\lambda}_{m n}} \iint_{\Omega} w^{0}(x, y) \varphi_{m n}(x, y) d x d y \\
& c_{2}=\frac{1}{\bar{\lambda}_{m n}} \iint_{\Omega} w^{1}(x, y) \varphi_{m n}(x, y) d x d y .
\end{aligned}
$$

Under expansion (12), the performance index (4) becomes

$$
\begin{aligned}
J_{N}[v(t)]= & \sum_{n=1}^{N} \sum_{m=1}^{M}\left[\mu_{1} W_{m n}^{2}\left(t_{f}\right)+\mu_{2}\left(\frac{d}{d t} W_{m n}\left(t_{f}\right)\right)^{2}\right] \\
& +\mu_{3} \int_{0}^{t_{f}} v^{2}(t) d t .
\end{aligned}
$$




\section{Control characterization}

The optimal control $v^{*}(t)$ in $U_{a d}$ is determined such that $J_{N}\left[v^{*}(t)\right]$ is minimum subject to (19)-(20). The necessary condition for the control $v(t)$ to be optimal is that $\delta_{v} J_{N}[v(t)]=$ 0 , and therefore,

$$
\begin{aligned}
\delta_{v} J_{N}[v(t)]= & \sum_{n=1}^{N} \sum_{m=1}^{M}\left[2 \mu_{1} W_{m n}\left(t_{f}\right) \delta W_{m n}\left(t_{f}\right)+\right. \\
& \left.2 \mu_{2} \dot{W}_{m n}\left(t_{f}\right) \delta \dot{W}_{m n}\left(t_{f}\right)\right] \\
& +2 \mu_{3} \int_{0}^{t_{f}} v(t) \delta v(t) d t=0 .
\end{aligned}
$$

That is

$$
\begin{aligned}
\delta_{v} J_{N}[v(t)]= & \sum_{n=1}^{N} \sum_{m=1}^{M}\left[\left(2 \mu_{1}\left(W_{m n}^{h}\left(t_{f}\right)+W_{m n}^{p}\left(t_{f}\right)\right) T_{1}\right.\right. \\
& \left.+2 \mu_{2}\left(\dot{W}_{m n}^{h}\left(t_{f}\right)+\dot{W}_{m n}^{p}\left(t_{f}\right)\right) T_{2}\right] \\
& +2 \mu_{3} \int_{0}^{t_{f}} v(t) \delta v(t) d t=0
\end{aligned}
$$

where

$$
\begin{aligned}
T_{1}= & \alpha \int_{0}^{t_{f}} e^{-\zeta \lambda_{m n}^{2}(t-\tau)} \sin \left(\bar{\lambda}_{m n}(t-\tau) \delta v(\tau) d \tau\right. \\
T_{2}= & \alpha \int_{0}^{t_{f}}\left[-\zeta \lambda_{m n}^{2} e^{-\zeta \lambda_{m n}^{2}(t-\tau)} \sin \left(\bar{\lambda}_{m n}(t-\tau)\right.\right. \\
& \left.+e^{-\zeta \bar{\lambda}_{m n}^{2}(t-\tau)} \bar{\lambda}_{m n} \cos \left(\lambda_{m n}(t-\tau)\right)\right] \delta v(\tau) d \tau,
\end{aligned}
$$

in which $\alpha=\Gamma_{1} \Gamma_{2} F_{1}\left(x_{1}, x_{2}\right) F_{2}\left(y_{1}, Y-2\right)$. This implies that

$$
\begin{aligned}
& 2 \alpha \mu_{1}\left(W_{m n}^{h}\left(t_{f}\right)+W_{m n}^{p}\left(t_{f}\right)\right)\left(e^{-\zeta \lambda_{m n}^{2}(t-\tau)} \sin \left(\bar{\lambda}_{m n}(t-\tau)\right)+\right. \\
& +2 \alpha \mu_{2}\left(\dot{W}_{m n}^{h}\left(t_{f}\right)+\dot{W}_{m n}^{p}\left(t_{f}\right)\right)\left[-\zeta \lambda_{m n}^{2} e^{-\zeta \lambda_{m n}^{2}(t-\tau)} \sin \left(\bar{\lambda}_{m n}(t-\tau)+\right.\right. \\
& \left.+e^{-\zeta \lambda_{m n}^{2}\left(t_{f}-\tau\right)} \cos \left(\bar{\lambda}_{m n}\left(t_{f}-\tau\right)\right)\right]+ \\
& +2 \mu_{3} v(\tau)=0
\end{aligned}
$$

Let

$$
\begin{aligned}
x & =\int_{0}^{t_{f}} e^{-2 \zeta \lambda_{m n}^{2}\left(t_{f}-\tau\right)} \sin \left(\bar{\lambda}_{m n}\left(t_{f}-\tau\right) \delta v(\tau) d \tau\right. \\
y & =\int_{0}^{t_{f}} e^{-2 \zeta \lambda_{m n}^{2}\left(t_{f}-\tau\right)} \cos \left(\bar{\lambda}_{m n}\left(t_{f}-\tau\right) \delta v(\tau) d \tau\right. \\
r_{1} & =\int_{0}^{t_{f}} e^{-2 \zeta \lambda_{m n}^{2}\left(t_{f}-\tau\right)} \sin ^{2}\left(\bar{\lambda}_{m n}\left(t_{f}-\tau\right) \delta v(\tau) d \tau\right. \\
r_{2} & =\int_{0}^{t_{f}} e^{-2 \zeta \lambda_{m n}^{2}\left(t_{f}-\tau\right)} \cos ^{2}\left(\bar{\lambda}_{m n}\left(t_{f}-\tau\right) \delta v(\tau) d \tau\right. \\
r_{3} & =\int_{0}^{t_{f}} e^{-2 \zeta \lambda_{m n}^{2}\left(t_{f}-\tau\right)} \sin \left(\overline { \lambda } _ { m n } ( t _ { f } - \tau ) \operatorname { c o s } \left(\lambda_{m n}\left(t_{f}-\tau\right) \delta v(\tau) d \tau\right.\right.
\end{aligned}
$$

By multiplying (24) first by $e^{-2 \zeta \lambda_{m n}^{2}\left(t_{f}-\tau\right)} \sin \left(\bar{\lambda}_{m n}\left(t_{f}-\tau\right)\right.$ and then by $e^{-2 \zeta \lambda_{m n}^{2}\left(t_{f}-\tau\right)} \cos \left(\bar{\lambda}_{m n}\left(t_{f}-\tau\right)\right.$ and integrate over $\left[0, t_{f}\right]$, we obtain the linear system

$$
\begin{aligned}
& d_{11} x+d_{12} y=e_{1} \\
& d_{21} x+d_{22} y=e_{2}
\end{aligned}
$$

where

$$
\begin{aligned}
d_{11} & =2 \mu_{1} \alpha^{2} r_{1}-2 \mu_{2} \alpha^{2} \zeta \lambda_{m n}^{2}\left(-\zeta \lambda_{m n}^{2} r_{1}+\bar{\lambda}_{m n} r_{3}\right)+2 \mu_{3} \\
d_{12} & =2 \mu_{2} \alpha^{2} \bar{\lambda}_{m n}\left(-\zeta \lambda_{m n}^{2} r_{1}+\bar{\lambda}_{m n} r_{3}\right) \\
d_{21} & =2 \mu_{1} \alpha^{2} r_{3}-2 \mu_{2} \alpha^{2} \zeta \lambda_{m n}^{2}\left(-\zeta \lambda_{m n}^{2} r_{3}+\bar{\lambda}_{m n} r_{2}\right) \\
d_{22} & =2 \mu_{2} \alpha^{2} \bar{\lambda}_{m n}\left(-\zeta \lambda_{m n}^{2} r_{3}+\bar{\lambda}_{m n} r_{2}\right) \\
e_{1} & =-2 \mu_{1} \alpha r_{1} W_{m n}^{h}-2 \mu_{2} \alpha r_{1} \dot{W}_{m n}^{h}\left(-\zeta \lambda_{m n}^{2} r_{1}+\bar{\lambda}_{m n} r_{3}\right) \\
e_{2} & =-2 \mu_{1} \alpha r_{1} W_{m n}^{h}-2 \mu_{2} \alpha r_{1} \dot{W}_{m n}^{h}\left(-\zeta \lambda_{m n}^{2} r_{1}+\bar{\lambda}_{m n} r_{2}\right)
\end{aligned}
$$

Upon solving system (26) for $x$ and $y$, and substituting these values into equation (24), an explicit solution for the optimal control $v(t)$ is readily obtained.

\section{Summary}

Active vibration control by the application of piezoelectric materials was applied to a thermally induced simply supported rectangular plate. Through a combination of Galerkin and variational approaches, the problem was set as an optimal control problem of a lumped parameter system and then the task of determining the corresponding displacement and velocity was reduced to just solving a set of ordinary differential equations.

\section{References}

1. S.S. Rao, M. Sunar, Analysis of distributed thermopiezoelectric sensors and actuators in advanced intelligent structures, J. AIAA, Vol. 31, 1280-1286, 1993.

2. Y. Ootao, Y. Tanigawa, Control of the transient thermoelastic displacement of a functionally graded rectangular plate bonded to a piezoelectric plate due to nonuniform heating, Act Mechanica, Vol. 148, 17-33, 2001.

3. T.R. Tauchert, F. Ashida, S. Sakata, Y. Takahashi, Control of temperature-induced plate vibrations based on speed feedback, Journal of Thermal Stresses, Vol. 29, 585-606, 2006.

4. I.F. Kirichok, Control of Axisymmetric vibrations and slef-heating of shells of revolution with piezoelectric sensors and actuators, International Applied Mechanics, Vol. 46, No. 8, 42-57, 2011.

5. I. Sadek, M. Abukhaled, Optimal control of thermoelastic beam vibrations by piezoelectric actuation, J. Control Theory and Applications, Vol 11, No. 3, 325-329, 2013.

6. J.C. Bruch, Jr, S. Adali, I.S. Sadek, J. M. Sloss, Structural control of thermoelastic beams for vibration suppression, Journal of Thermal Stresses, Vol. 16, No. 3, 249-263, 1993.

7. M. I. Friswell, D.J. Inman, R.W. Rietz, Active damping of thermally induced vibrations, Journal of Intelligent Material Systems and Structures, Vol. 8, No.8, 678685, 1997.

8. N.G. Sharnappa, R. Sethuraman, Thermally induced vibrations of piezo-thermo-viscoelastic composite beam with relaxation times and system response, Multidiscipline Modeling in Materials and Structures, Vol. 6, No. 1, 120-140, 2010. 